Revue

Revue de l'histoire des religions

de Ihistoire des religions

Bruce LINCOLN, Apples and Oranges. Explorations in, on and with Comparison

Chicago, The University of Chicago Press, 2018

Ron Naiweld

\title{
CpenEdition
}

Journals

Édition électronique

URL : http://journals.openedition.org/rhr/11033

DOI : 10.4000/rhr.11033

ISSN : 2105-2573

Éditeur

Armand Colin

Édition imprimée

Date de publication : 1 mars 2021

Pagination : 140-142

ISBN : 978-2-200-93375-3

ISSN : 0035-1423

Référence électronique

Ron Naiweld, "Bruce Lincoln, Apples and Oranges. Explorations in, on and with Comparison ", Revue de I'histoire des religions [En ligne], 1 | 2021, mis en ligne le 19 mars 2021, consulté le 31 mars 2021. URL : http://journals.openedition.org/rhr/11033; DOI : https://doi.org/10.4000/rhr.11033

Ce document a été généré automatiquement le 31 mars 2021.

Tous droits réservés 


\section{Bruce LinColN, Apples and Oranges. Explorations in, on and with Comparison}

Chicago, The University of Chicago Press, 2018

Ron Naiweld

\section{RÉFÉRENCE}

Bruce Lincoln, Apples and Oranges. Explorations in, on and with Comparison, Chicago, The University of Chicago Press, 2018, 368 p., 22,9 cm, 35 \$, ISBN 978-0-226-56407-4.

1 Professeur émérite en histoire des religions à la Divinity School de l'Université de Chicago, Bruce Lincoln regroupe ici plusieurs études, la plupart des années 2010, sur la pratique de la comparaison des phénomènes religieux, historiques, discursifs et sociaux. Les études relèvent des compétences de l'auteur et mobilisent des sources perses et grecques de l'époque classique et des sources européennes du Moyen Âge. Le livre est divisé en quatre parties dont la première, "General Observations », est programmatique. L'auteur donne sa vision de la méthode comparatiste, ses recommandations et ses avertissements. Il défend principalement une comparaison "faible ", qui se focalise sur des phénomènes spécifiques et ne cherche pas à comparer des cultures et des civilisations dans leur ensemble. Il encourage aussi les comparatistes à ne pas se contenter des similitudes au niveau des signes, mais de creuser les contextes particuliers dans lesquels ces signes ont été forgés.

2 Pour bien montrer les dangers que courent les comparatistes qui n'observent pas ces règles, B. Lincoln consacre la deuxième partie du livre à la critique de deux comparatistes, Carlo Ginzburg et Michael Witzel. L'auteur fait les louanges de la méthode comparatiste prudente adoptée par Ginzburg dans sa dissertation de 1964, mais il critique fortement sa Storia notturna (1989) où Ginzburg « exchanged a modest, carefully delimited, and highly successful comparative method for a looser, riskier, 
more open-ended style whose ambitions were more grandiose ». La critique est moins mitigée dans le chapitre suivant sur le livre de Witzel The Origins of the World's Mythologies (2012). Non seulement Witzel, professeur de sanskrit à Harvard, mènerait dans le livre une comparaison générale et trop poussée au lieu de se contenter de la comparaison faible, mais il le ferait en s'appuyant sur des ethnologues allemands associés à la Kulturkreislehre, dont Herman Baumann. Lincoln reconnaît que Witzel " does not follow Baumann where the latter's racism is most obvious and offensive, but he rarely identifies it as such or rejects it outright». Lincoln utilise l'exemple pour avertir les comparatistes de toujours prendre en considération le contexte politique et social dans lequel les comparaisons sont faites et présentées.

3 Les derniers chapitres sont des études qui démontrent les avantages de la méthode comparatiste offerte par l'auteur. Au-delà de l'intérêt que présente chacun des chapitres, le livre est intéressant pour sa vision de la méthode comparatiste surtout quand elle est utilisée sur des faits et des phénomènes religieux. Dans le texte qui suit l'introduction, coécrit avec Cristiano Grottanelli et publié pour la première fois en 1985, les deux auteurs tracent une petite histoire de la discipline «History of Religions » et expriment leurs prévisions et leurs souhaits quant à son avenir comparatiste. Ils distinguent cinq mythic ancestors : Karl Marx, Friedrich Engels, Émile Durkheim, Max Weber et Bronisław Malinowski. Les cinq sont unis par trois facteurs : 1 / Ils situent les phénomènes religieux dans un contexte social large ; 2/ à l'exception de Malinowski, ils emploient tous des méthodes comparatistes ; 3 / à l'exception de Durkheim, ils sont tous sensibles aux dynamiques des phénomènes religieux et sociaux, leurs évolutions et leurs transformations.

La contribution de chaque "père » est décrite brièvement. Marx est celui qui a offert " a rich and suggestive treatment of religion as a mode of ideology ». Engels, contrairement à Marx, n'a pas qualifié la religion de fausse conscience tout court car pour lui les mouvements et les attitudes religieux pouvaient exprimer et maintenir des tensions sociales réelles. Weber a montré que la religion n'est pas secondaire ou périphérique par rapport à la structure sociale mais qu'elle peut en être la cause. Durkheim et son école "focused on the ways in which religious phenomena [...] fostered both a common worldview and powerful sentiments of solidarity among those who shared them». Enfin, Malinowski a partagé avec Durkheim sa vision fonctionnaliste de la religion, mais contrairement au sociologue français et à son école qui étudiaient la religion comme l'expression des systèmes universels de pensées et de sentiments, il a mis l'accent sur les manières spécifiques par lesquelles la religion fonctionnait dans des sociétés particulières.

5 Le choix et la présentation des ancêtres de la discipline expriment bien, non seulement la démarche du livre dans son ensemble, mais aussi ses limites. Le fait que les cinq viennent tous de la partie judéo-chrétienne de notre civilisation ne semble pas constituer ici un problème. B. Lincoln ne l'explique pas d'ailleurs dans le livre. Cela va à l'encontre de la méthode prudente qu'il avance et qui déconstruit la neutralité affichée des systèmes symboliques, des théories et des idéologies surtout quand elles sont données sous la forme d'un mythe. Or, on constate ici un silence à l'égard des origines religieuses qui avaient influencé les manières dont les fondateurs de la discipline ont abordé les phénomènes religieux. L'usage neutre de la catégorie de " religion » dans un discours savant de ce type est peut-être attendu d'un texte publié en 1985. Il l'est un peu moins pour un livre programmatique sur la comparaison en histoire des religions 
publié en 2018. Dans l'espace entre les deux publications notre usage de la catégorie a été plusieurs fois mis en question, comme dans le livre de Talal Asad, Genealogies of Religion: Discipline and Reasons of Power in Christianity and Islam (Johns Hopkins University Press, 1993) ou encore le livre de Carlin A. Baton et Daniel Boyarin, Imagine no Religion. How Modern Abstraction hide Ancient Realities (Fordham University Press, 2016).

Le lecteur peut rester sur sa faim par rapport à la posture individuelle de B. Lincoln à partir de laquelle ses comparaisons et leur présentation sont pratiquées. Un message fort présent dans le livre est que les récits des poètes et des historiens portent souvent une vision idéalisée ou déformée (parfois les deux) des rapports sociaux et politiques auxquels sont soumis les auteurs. En effet, dans l'introduction du livre, B. Lincoln donne quelques détails biographiques sur l'évolution de sa démarche scientifique mais on ne peut pas dire qu'il leur accorde le même traitement que pour ses autres sources. $\mathrm{Au}$ cours du livre, on n'a aucun renseignement sur les conditions économiques, institutionnelles et personnelles dans lesquels il a produit et pensé ses comparaisons. Le refoulement très commun de cette dimension de l'activité scientifique aide à maintenir l'image neutre du regard comparatiste. Mais en dehors du livre, l'illusion se révèle en tant que telle. La réalité contemporaine introduit des différences importantes au sein de la communauté savante, ce qui fragilise l'image idéologique portée par le livre d'une posture partagée par toutes celles et tous ceux qui pratiquent la comparaison. Les mouvements migratoires de nos sociétés et la mixité ethnique, sociale et religieuse du public universitaire font que l'avenir de la discipline sera sans doute comparatiste, comme l'est déjà son présent, mais pour que les comparaisons soient pertinentes pour l'histoire et pour nos façons de l'étudier, il faudra introduire dans l'analyse des aspects particuliers de l'activité du chercheur ou de la chercheuse et mieux prendre en compte les conditions historiques réelles de la comparaison.

\section{AUTEURS}

\section{RON NAIWELD}

CNRS, Centre de Recherches Historiques, Paris. 